\title{
The upgrade of the ATLAS silicon strip tracker
}

\author{
Jose Bernabeu* \\ On behalf of the ATLAS Collaboration \\ IFIC - Instituto de Fisica Corpuscular (University of Valencia and CSIC), \\ Edificio Institutos de Investigacion, Apartado de Correos 22085, E-46071 Valencia, Spain \\ E-mail: Pepe.Bernabeu@ific.uv.es
}

\begin{abstract}
A phased upgrade of the Large Hadron Collider (LHC) at CERN is planned. The last upgrade phase (HL-LHC) is currently foreseen in 2022-2023. It aims to increase the integrated luminosity to about ten times the original LHC design luminosity. To cope with the harsh conditions in terms of particle rates and radiation dose expected, the ATLAS collaboration is developing technologies for a complete tracker replacement. This new detector will need to provide extreme radiation hardness and a high granularity, within the tight constraints imposed by the existing detectors and their services. An all-silicon high-granularity tracking detector is proposed. An international R\&D collaboration is working on the strip layers for this new tracker. A number of large area prototype planar detectors produced on p-type wafers have been designed and fabricated. Several prototype modules using prototype readout electronics have been produced, as well as multi-module assemblies. They have allowed testing of many system aspects, including different powering options. This presentation shows the proposed new tracker and the design of the strips layers. The module assembly for the strip tracker is described, and results from silicon sensors before and after irradiation are shown. Status of the project and performance of module assemblies are summarised.
\end{abstract}

The 21 st International Workshop on Vertex Detectors

16-21 September 2012

Jeju, Korea

*Speaker. 


\section{Introduction}

The Large Hadron Collider (LHC) is providing proton-proton collisions to the different experiments. Physics data are being recorded by the ATLAS experiment [1], which is designed to fully exploit the phyics potential of the LHC. A phased upgrade of the LHC is planned with a continuous increase in the delivered luminosity (Fig.1). The last upgrade (Phase 2) requires an upgraded machine called High Luminosity LHC (HL-LHC). With a target of $\sim 3000 \mathrm{fb}^{-1}$ total recorded integrated luminosity per experiment. The HL-LHC will allow to improve the precision in different measurements within the Standard Model, enlarge the discovery region of particles predicted by some supersymmetric theories and enhance the sensitivity to low-rate phenomena inaccessible at the LHC [2].

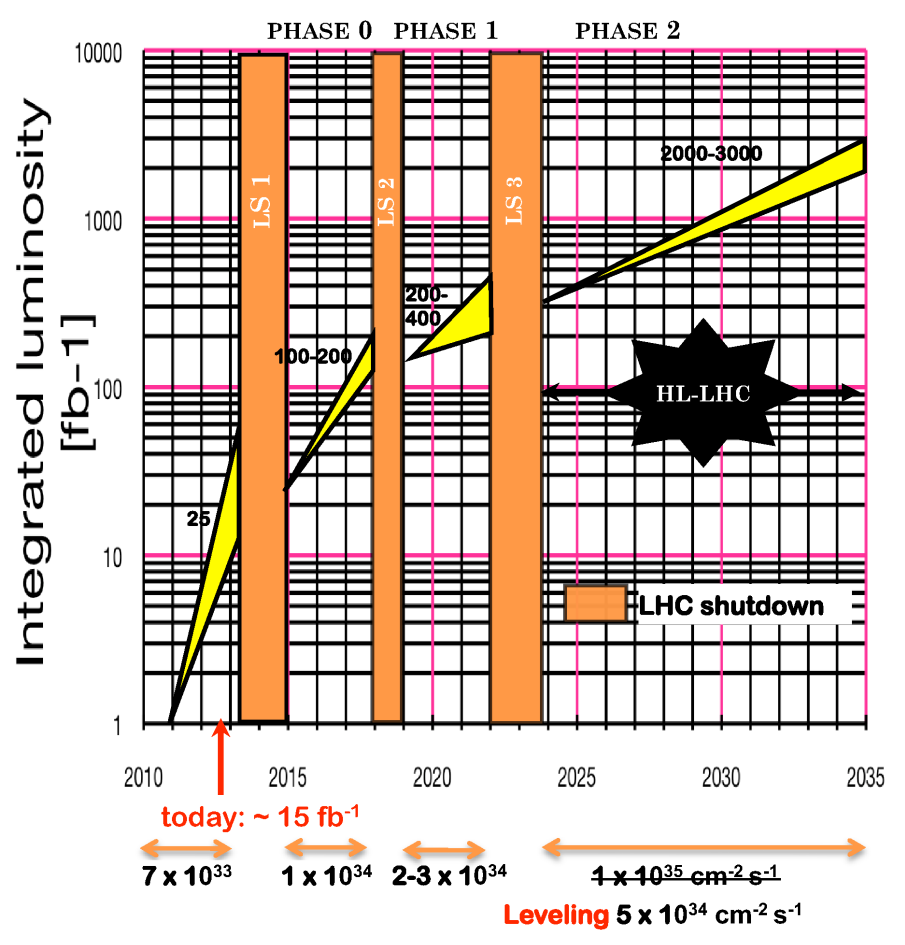

Figure 1: Forecast of the luminosity upgrade with indication of "today" as September 20, 2012.

\subsection{New ATLAS Inner Tracker}

The expected luminosity at the HL-LHC will increase the number of pile-up interactions per crossing from $\sim 23$ (as predicted in the current LHC at the design luminosity) up to $\sim 140$. The current tracker will not be able to stand the cumulated radiation damage and it will not provide the required tracking performance due to the huge increase in the channel occupancy. Therefore, a completely new inner tracker must be designed and built for the HL-LHC operation. The two main requirements are: on the one side much better granularity in order to be able to distinguish the tracks of the different particles, on the other side it needs to have much better radiation hardness.

Most probably, the future ATLAS tracker will be an all silicon-based system. Figure 2 shows the current Letter-Of-Intent layout [3]. The central barrel region consists of four pixel layers and 
five strip layers plus an additional stub. The stub is a layer with only strip sensors in the last $10 \mathrm{~cm}$ at each end. The three innermost strip layers have short strips $(2.4 \mathrm{~cm}$ long) and the outer two strip layers and the stub have long strips $(4.8 \mathrm{~cm}$ long). The forward region is covered by six pixel and seven strip disks. This layout ensures 14-hit coverage in the pseudo-rapidity range $|\eta| \leq 2.5$.

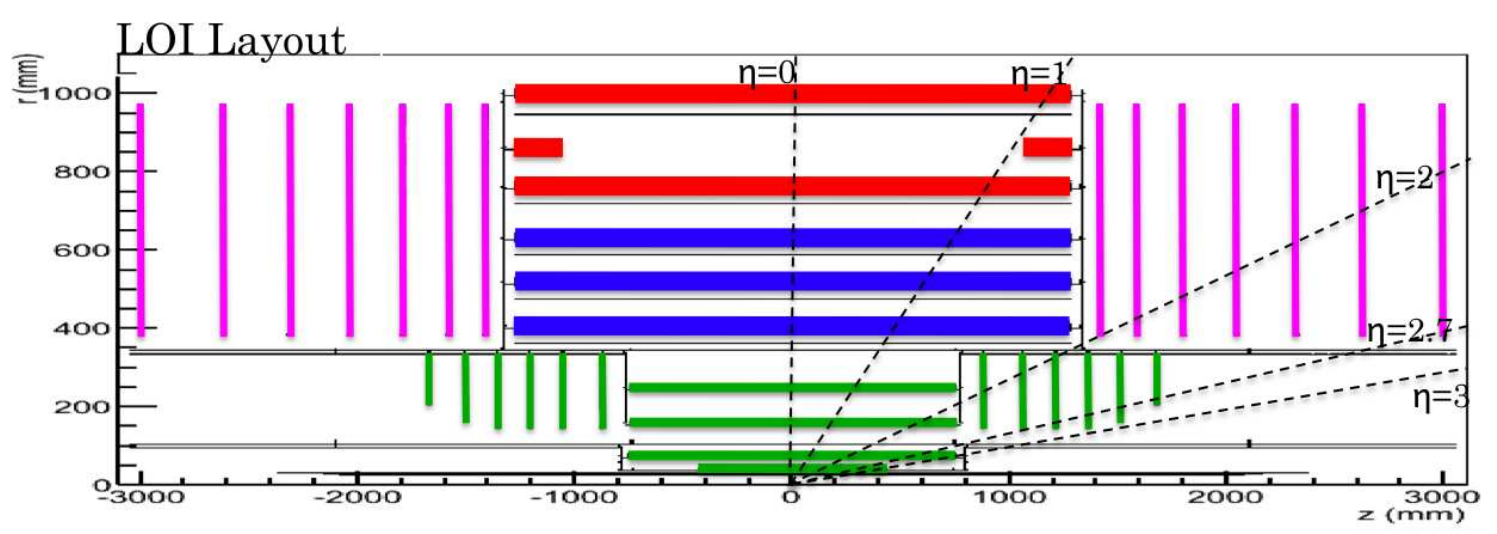

Figure 2: LoI layout for the future ATLAS tracker.

Figure 3 shows the expected fluence map at a given radial distance to the beamline and along the beamline for the LoI layout and a total integrated luminosity of $3000 \mathrm{fb}^{-1}$. The maximum expected fluence in the strip barrel area is $1 \times 10^{15} \mathrm{n}_{e q} / \mathrm{cm}^{2}$, corresponding to the innermost short strip layer. This number includes a safety factor of two. The first barrel long-strip layer expects $4.7 \times 10^{14}$ $\mathrm{n}_{e q} / \mathrm{cm}^{2}$. For the strip forward region, the maximum expected fluence is $1.2 \times 10^{15} \mathrm{n}_{e q} / \mathrm{cm}^{2}$.

\section{$1 \mathrm{MeV}$ neutron equivalent fluence}

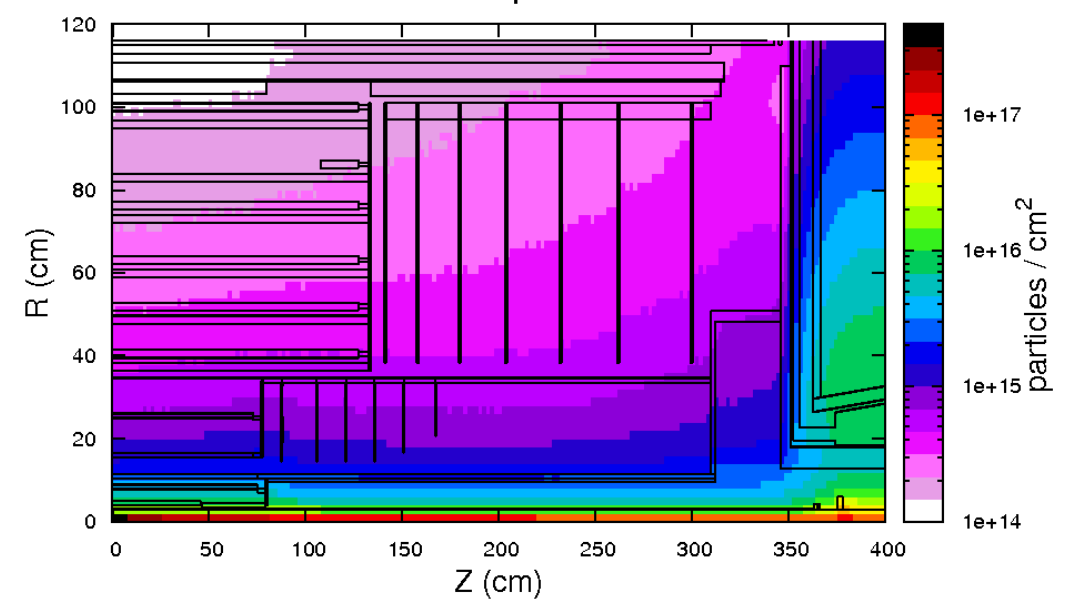

Figure 3: Expected fluence map for $3000 \mathrm{fb}^{-1}$.

\section{Strip Tracker Design}

Several R\&D projects are well advanced in terms of design and assembly of strip modules integrating sensors, hybrids with readout electronics, cooling and services. Figure 4 shows the 
baseline and fallback integration concepts for the short-strip barrel region. In the baseline stave concept [4], a common mechanical structure integrates the sensors, the electrical lines (bus cable) and cooling circuit. The stave has a central core composed of a spacing material (carbon-foam or honeycomb) and carbon fiber facings glued on both sides. Silicon microstrip detectors are glued on the bus cable and hybrids carrying the front-end electronics are then glued on top of the sensitive side of the detectors. In the fallback super-module concept [5], individual double-sided silicon strip modules are assembled into a light-weighted local support structure. Each module is composed of a central base-board, two silicon sensors and four hybrids with readout electronics.

Prototypes, in both options, of modules with prototype electronics, sensors and assembly components have been constructed and electronically tested. Assemblies of modules in short staves or supermodules $(40 \mathrm{~cm}$ instead of the final $120 \mathrm{~cm}$ ) have been produced and tested.

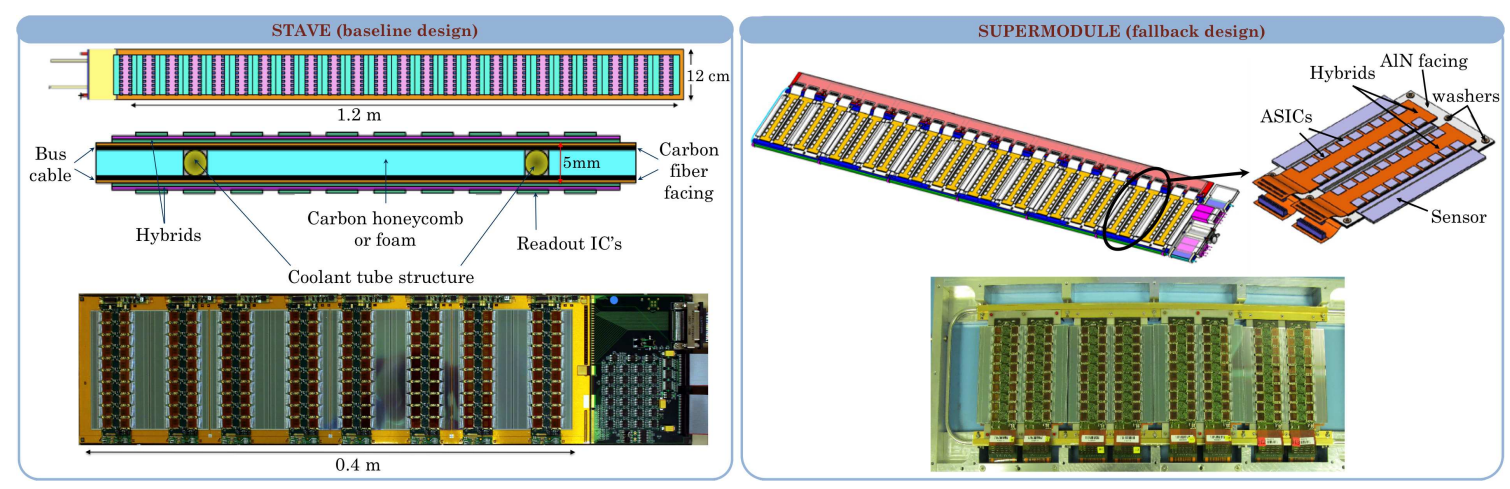

Figure 4: Stave (left) and super-module (right) barrel strip integration concepts.

\section{Silicon Sensors}

Silicon sensors for the new tracker have been designed. The choice of $\mathrm{n}^{+}$strips in $\mathrm{p}$ type substrate was found to be most adequate. With n-strips, electrons are collected, thus, the signal is faster and reduces the effect of trapping centres produced by the high level of radiation. P-type substrate permits to have a single sided process during manufacturing, a major advantage compared to having a $\mathrm{n}$-type substrate (in the $\mathrm{n}^{+}$-in-n option) which requires expensive manufacture processing on both sides. In addition, the selected $\mathrm{n}^{+}$-in-p option depletes always from the strip side, permitting to work underdepleted.

A large area $\mathrm{n}^{+}$-in-p silicon strip sensor has been developed by the ATLAS collaboration and produced by Hamamatsu Photonics in a 6-inch $(150 \mathrm{~mm})$ wafer [6]. The mask layout of the sensor is shown in Fig. 5. The detector area is $97.54 \times 97.54 \mathrm{~mm}^{2}$ and its thickness is $320 \mu \mathrm{m}$. The bulk is p-type float zone silicon with a lattice orientation $\left\langle\begin{array}{llll}1 & 0 & 0\end{array}\right\rangle$. The $\mathrm{n}^{+}$readout strips are AC-coupled and have polysilicon bias resistors. The isolation structure is p-stop. The sensor has four $2.39 \mathrm{~cm}$ strip segments, two with axial strips parallel to the sensor edges and two with stereo strips tilted at an angle of $40 \mathrm{mrad}$. The reason of this tilt is that when sensors are assembled back to back, an axial strip hit on one side matches a stereo strip hit on the other side, giving not only $\phi$ information, but also on the longitudinal coordinate, $\mathrm{Z}$. The strip pitch is $74.5 \mu \mathrm{m}$ and there are a total of 1280 strips per segment. 


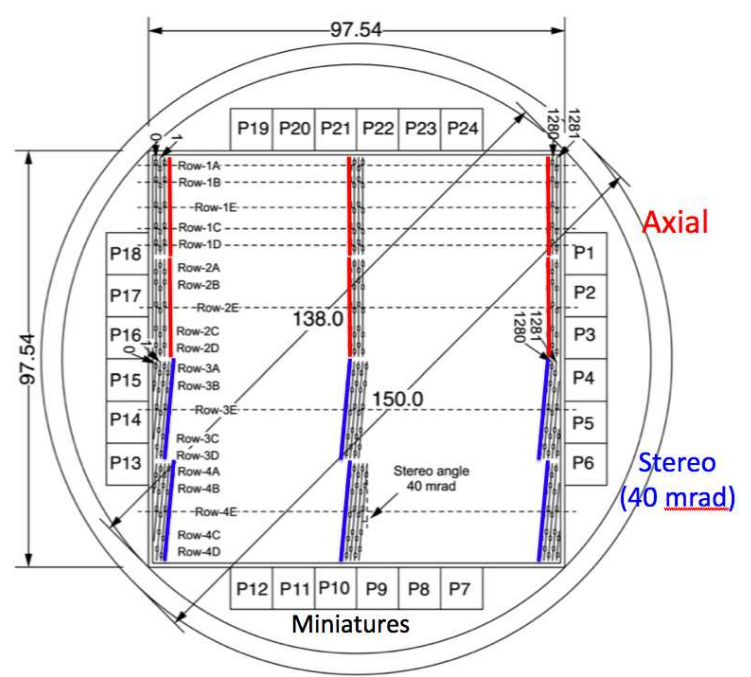

Figure 5: Mask layout of the barrel strip sensor prototypes.

In addition to the main sensor, $2410 \times 10 \mathrm{~mm}^{2}$ miniature sensors are included in the wafer. The miniature sensors have 104 strips of $8 \mathrm{~mm}$ length and implement different surface strip isolation structures (p-stop) and punch-through protection (PTP) structures.

\section{Measurements on Full Size Sensors}

The full size sensors have been extensively tested before irradiation. Both the bulk and strip characteristics have been thoroughly evaluated to check the sensors were effectively compliant with the required technical specifications before irradiation [7]. Figure 6 shows the comparison of the different specifications and the measured parameters, all within specifications. As an example, the depletion voltage measurement is shown.

\begin{tabular}{|l|c|c|}
\hline Parameter & Specification & Measurement \\
\hline Leakage Current & $<200 \mu \mathrm{A} @ 600 \mathrm{~V}$ & $200 \mathrm{nA}-370 \mathrm{nA}$ \\
\hline Depletion Voltage & $<500 \mathrm{~V}$ & $190 \mathrm{~V}-245 \mathrm{~V}$ \\
\hline Interstrip Capacitance & $<1.1 \mathrm{pF} / \mathrm{cm}$ (3probes) & $0.7 \mathrm{pF} / \mathrm{cm}$ \\
\hline Coupling Capacitance & $>20 \mathrm{pF} / \mathrm{cm}$ & $24-30 \mathrm{pF} / \mathrm{cm}$ \\
\hline Polysilicon Resistance & $1.5 \pm 0.5 \mathrm{M} \Omega$ & $1.3-1.6 \mathrm{M} \Omega$ \\
\hline Interstrip Resistance & $>10 \mathrm{xRbias} \approx 15 \mathrm{M} \Omega$ & $>19 \mathrm{G} \Omega$ \\
\hline
\end{tabular}

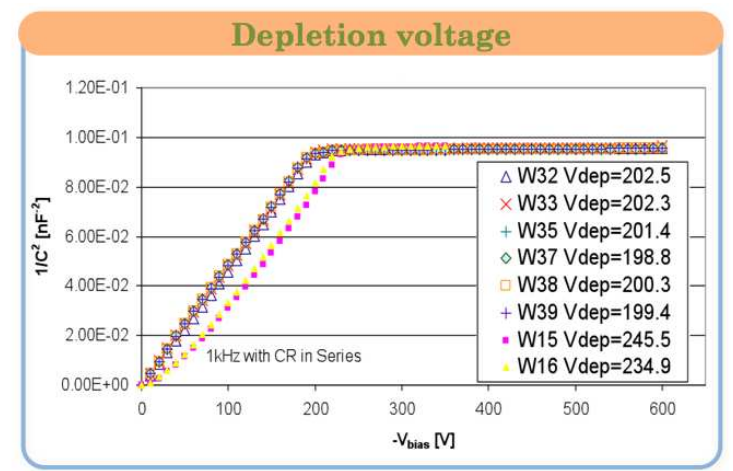

Figure 6: Values of the pre-radiation full size sensor specifications and measurements (left). Depletion voltage measurement for different sensors (right).

Modules for the two design concepts have been built with these full size sensors. Assembled with prototype electronics, calibration tests show good module performance with $\sim 600$ electron noise for both design concepts. One module was irradiated in the PS facility at CERN, the $24 \mathrm{GeV}$ 
proton beam scanned the full module area. The module was biased, powered and clocked during the irradiation up to $1.9 \times 10^{15} \mathrm{n}_{e q} / \mathrm{cm}^{2}$, well above the expected dose. After irradiation the module was fully functional and the noise slightly increased by $10 \%$, well in agreement with predicted electronic simulations.

\section{Measurements on Miniature Sensors}

Miniature sensor have been mainly used for irradiation studies. They have been irradiated with protons, pions and neutrons at different irradiation facilities. The doses were all up to at least $1 \times 10^{15} \mathrm{n}_{e q} / \mathrm{cm}^{2}$, in a few irraditions the sensors were irradiated up to $1 \times 10^{16} \mathrm{n}_{e q} / \mathrm{cm}^{2}$. The IV-characteristics, the depletion voltage, the interstrip capacitance and resistance and the charge collection efficiency have been measured [8].

The irradiated sensors were distributed among different testing laboratories to check the charge collection. Very good agreement was found in the measurements among the different samples (Fig. 7), which is a good indication for the measurement validity as each laboratory uses a complete different data acquisition system. A signal to noise $(\mathrm{S} / \mathrm{N})$ ratio of $\sim 15$ was found after $1 \times 10^{15}$ $\mathrm{n}_{e q} / \mathrm{cm}^{2}$ irradiation with $500 \mathrm{~V}$ of sensor bias, well above the required specification of 10 . Collected charge as function of the fluence show a good safety margin (Fig. 7). Also, it is worth to notice the good agreement of results among the different particle irradiations, which verifies the NIEL equivalences.
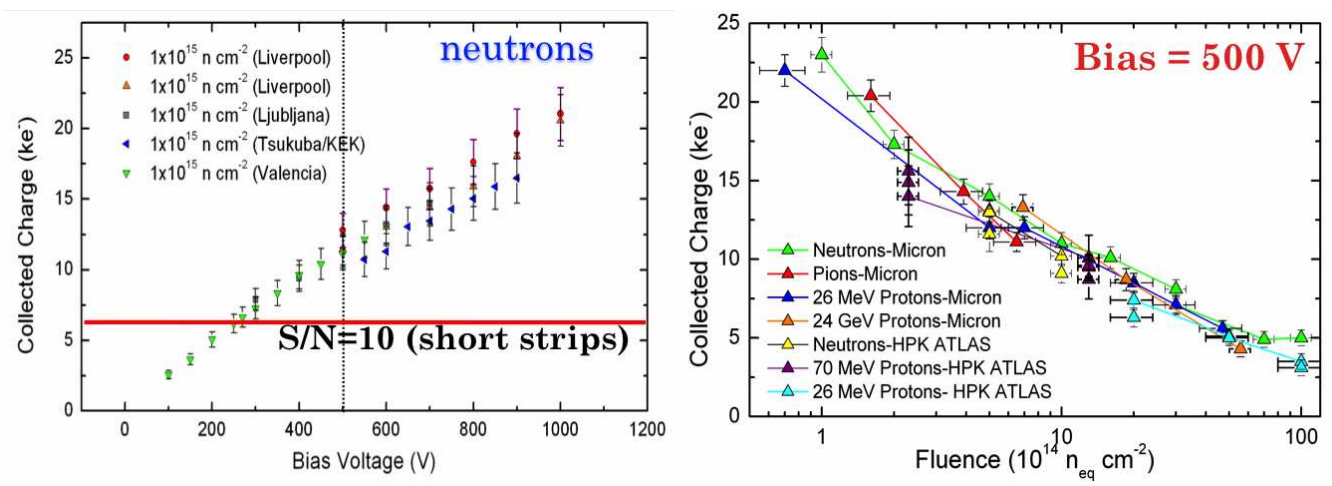

Figure 7: Collected charge of neutron irradiated miniature sensors as a function of the bias voltage (left). $\mathrm{S} / \mathrm{N}$ is above specification. Collected charge of irradiated miniature sensors in different facilities as function of the fluence (right).

Miniature sensors have also been used to test the so-called punch-through protection (PTP) structure and for observation of charge multiplication in heavily irradiated sensors $\left(>5 \times 10^{15} \mathrm{n}_{e q} / \mathrm{cm}^{2}\right)[9$, $10,11]$.

\section{Barrel stave powering}

As mentioned, the integration stave concept uses a multi-module structure. One stave hosts 13 identical modules per side. There are two hybrids in a short strip module, hosting the readout 
and control electronics necessary for the readout of the 5120 channels. Each hybrid includes 20 prototype ASICs (ABCN-25 [12]), each with 128 channels and binary readout.

This new silicon strip tracker occupies a bigger volume $\left(192 \mathrm{~m}^{2}\right)$ and has much higher granularity (74 million strip channels) than the present one. Thus, using an individual power cable per module is not possible in the very restricted space for the tracker services. In addition, the tracker cables in ATLAS are $\sim 100 \mathrm{~m}$ long to the counting room. The power loss along the cable is not negligible.

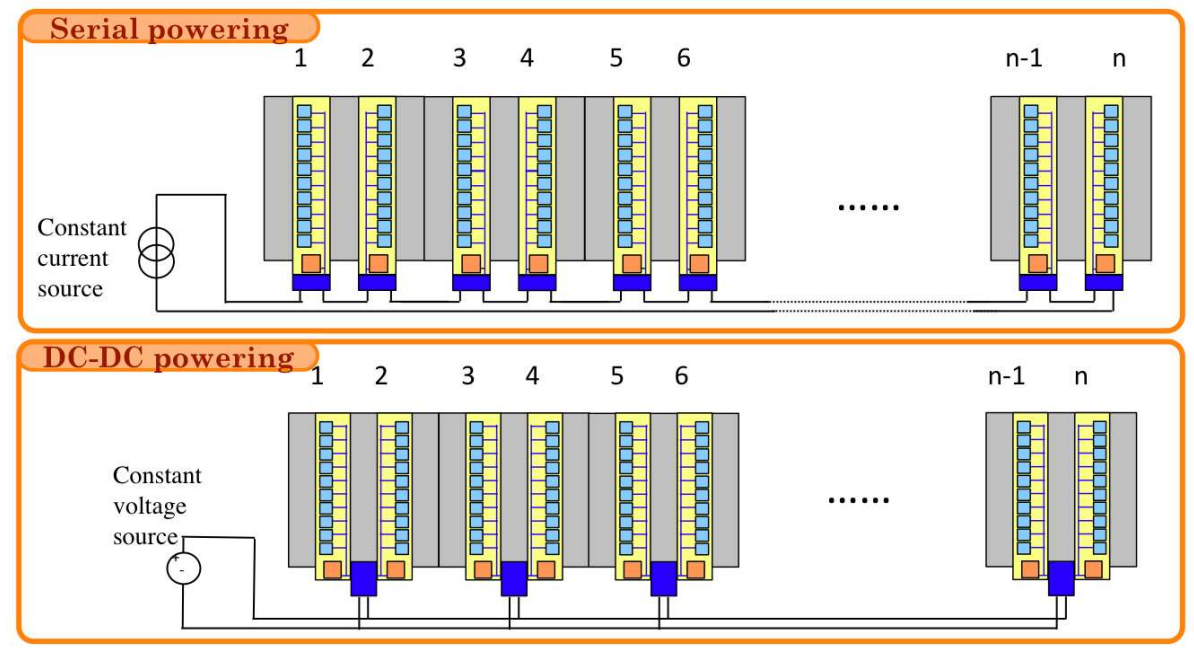

Figure 8: Serial and DC-DC powering architectures.

Two power architectures have been considered for the staves: serial powering and DC-DC powering (Fig. 8). In serial powering, a constant current source provides power to all the modules of a stave side with a shunt regulator circuit and a single power cable. The current is constant along the chain, and is equal to the current required by an individual hybrid or module. The lower current in a single cable minimises the power losses in the cable. There are as many voltage steps as elements in the chain. Another approach is used in DC-DC powering, in which a constant voltage source provides power to the modules after one or several voltage conversion steps made by buck DC-DC converters. It also requires a single power line. It uses a much higher voltage at the source $(60-80 \mathrm{~V})$ with much lower current. A first voltage conversion at the stave end reduces the voltage to $10-12 \mathrm{~V}$ providing more current. A final voltage conversion at the module supplies the required voltage and current to the electronics.

Strong R\&D efforts have been dedicated by the collaboration to the study and development of both powering architectures, each with advantages and drawbacks. The program included the construction of stavelets, which are multi-module prototypes with four modules, instead of 13 modules like the final staves will have.

The edges of the stavelets have been widened to provide space to attach the power and protection components on one edge, for both powering architectures (Fig. 9). Testing of these stavelets showed very similar electrical behaviour for both stavelets. The overall output noise in the multimodule stavelet arrangement is increased by approximately 20 electrons with respect to the noise values of the same modules in individual test frames before the stavelet assembly [4]. 

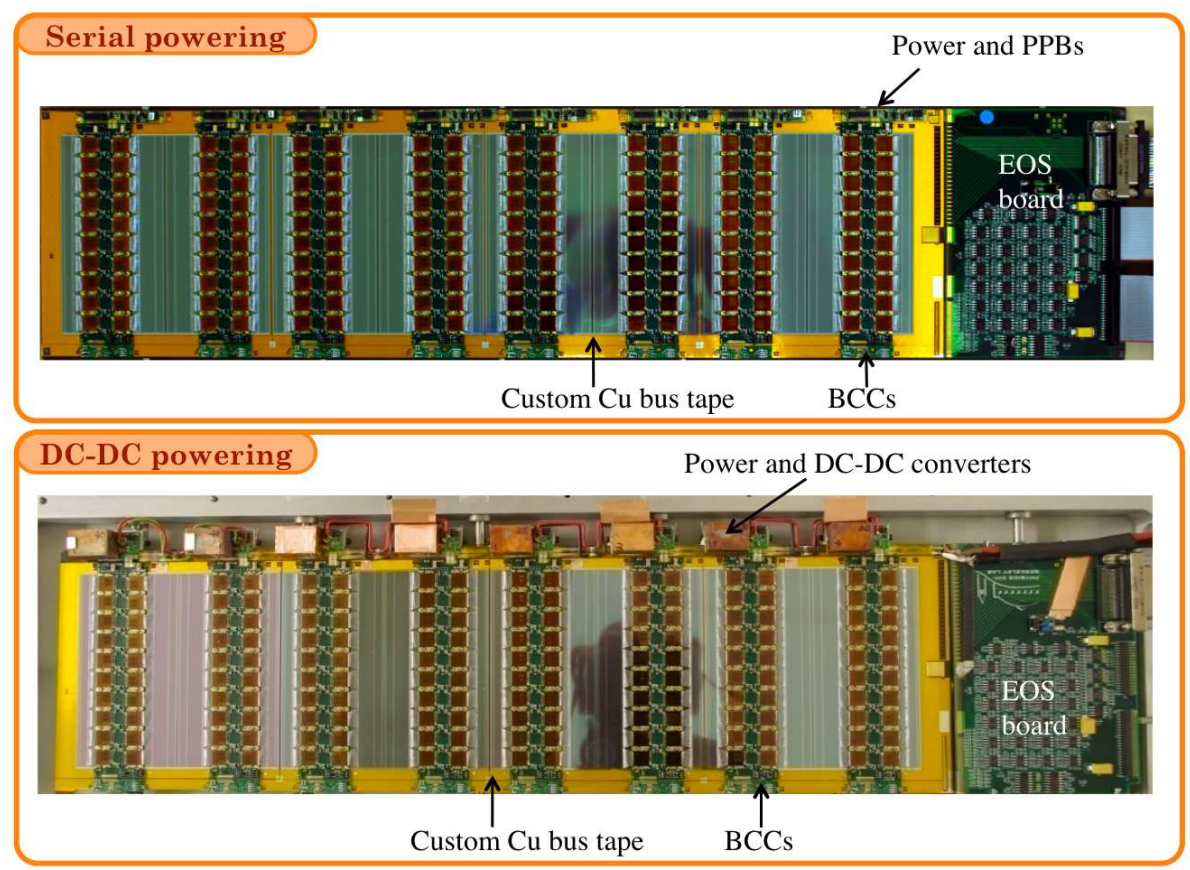

Figure 9: Serial and DC-DC stavelets.

\section{Endcap petals}

The endcap disks will be composed of 32 petals. The petal is the multi-module structure in the endcap region and follows very closely the design of the barrel staves. However, the design of the petal is more complex in comparison to the barrel. The geometry is complicated as it needs six rings with six different sensors (Fig. 10). Each petal hosts 9 modules and each one is different.
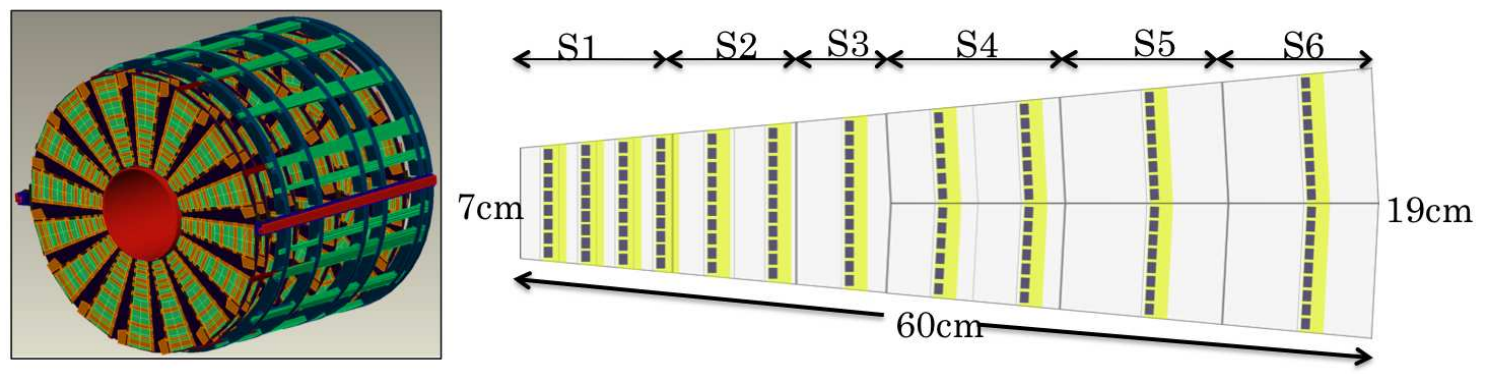

Figure 10: Endcap disks and petal geometry.

Due to this increased complexity, the prototyping stage of the petals is not as mature as in the barrel. A first prototype of a petal assembly is being carried out, the so-called petalet. The petalet consists of three small modules in a specific endcap configuration that is not present in the barrel stave (Fig. 11). It uses cheaper 4-inch wafer sensors and include some possible endcap specificities, such as truncated strips or embedded fanins.

The petal design has the strips pointing to the center of the disk. There should be a stereo angle between the strips in the front and back sides of the petal. In order to keep both sides the same, 

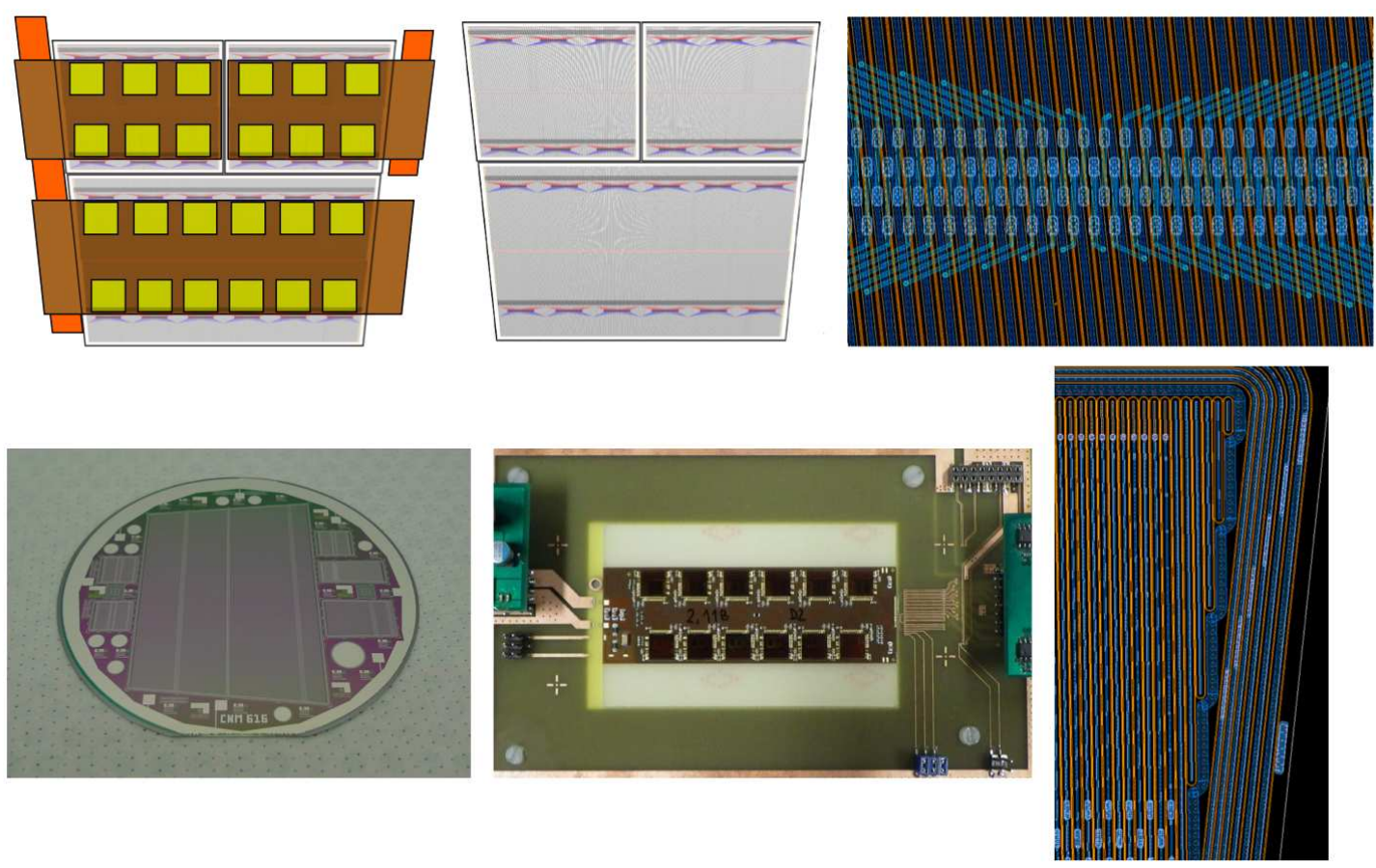

Figure 11: Petalet and components. Petalet configuration (top left and top center), the sensors (bottom left) include embedded fanins (top right) and truncated strips at the edges (bottom right). First hybrid is tested (bottom center).

the strips in each side are tilted half of the stereo angle. Thus, the strips are not exactly pointing to the center of the disk. In one of the design options, the sensors have truncated strips at the edge (Fig. 11). This arrangement will be tested in the petalet.

The sensors have been fabricated, delivered and tested. The first hybrid type has been produced and tested successfully. Work on the rest of the components, carbon fibre structure, bus tape, powering, is also progressing well.

\section{Conclusions}

The present ATLAS tracker will be replaced by a new all-silicon tracker. Sensors for this tracker have been produced in $\mathrm{n}^{+}$-in-p technology. The performance is very good and final design specifications are already fullfilled. Miniature sensors have been produced and used to verify radiation hardness. Modules and stavelets have been constructed with these sensors, achieving very good performance. Sensors and modules are fully functional after irradiation at the expected dose in HL-LHC. Two powering architectures have been verified with multi-module assemblies. Significant progress has also been achieved on the endcap module prototypes, with a petalet expected to be built and tested in the following months.

\section{References}

[1] The ATLAS Collaboration, The ATLAS Experiment at the CERN Large Hadron Collider, JINST 3 , S08003, 2008. 
[2] K. Jakobs, Physics at the LHC and sLHC, Nucl. Instr. and Meth. A 636 (2011) 1-7.

[3] A. Clark et al., ATLAS Phase II Letter of Intent: Backup Document, ATL-UPGRADE-PUB-2012-004 (2012) $12 \mathrm{pp}$.

[4] S. Díez, Silicon strip staves and petals for the ATLAS Upgrade tracker of the HL-LHC, Nucl. Instr. and Meth. A 699 (2013) 93-96.

[5] S. Gonzalez-Sevilla et al., Electrical performance of a silicon micro-strip super-module prototype for the High-Luminosity LHC collider, Nucl. Instr. and Meth. A 699 (2013) 102-106.

[6] Y. Unno et al., Development of n-in-p silicon sensors for very high radiation environments, Nucl. Instr. and Meth. A 636 (2011) 24-30.

[7] J. Bohm et al., Evaluation of the bulk and strip characteristics of large area $n$-in-p silicon sensors intenteded for a very high radiation environment, Nucl. Instr. and Meth. A 636 (2011) 104-110.

[8] K. Hara et al., Testing of bulk radiation damage of n-in-p silicon sensors for very high radiation environments, Nucl. Instr. and Meth. A 636 (2011) 83-89.

[9] J. Bernabeu, Silicon Strip Detectors for the ATLAS HL-LHC Upgrade, in proceedings of $10^{\text {th }}$ International Conference on Large Scale Applications and Radiation Hardness of Semiconductor Detectors, 6-8 July, 2011, Florence, PoS (RD11) 021.

[10] S. Lindgren et al., Testing of surface properties pre-rad and post-rad of $n$-in-p silicon sensors for very high radiation environments, Nucl. Instr. and Meth. A 636 (2011) 111-117.

[11] G. Casse et al., Evidence of enhanced signal response at high bias voltages in planar silicon detectors irradiated up to $2.2 \times 10^{16} \mathrm{neq}_{\text {eq }} \mathrm{cm}^{2}$, Nucl. Instr. and Meth. A 636 (2011) 56-61.

[12] W. Dabrowski et al., Design and performance of the ABCN-25 readout chip for ATLAS inner detector upgrade, IEEE Nucl.Sci.Symp.Conf.Rec.(2009) 373-380. 